\title{
Efficient Analytical Model for Calculation of the Influence Zone inside the Subsoil below Foundations Slabs
}

\author{
P. Kuklík, M. Kopáčková
}

\begin{abstract}
This paper presents analytical approaches for evaluating the depth of the influence zone. The model focuses on variations in the ground water table. Referring to [6], the term influence zone is assigned to the region where the load has a substantial influence on the deformation of the soil skeleton. The first approach relies on an analytical solution based on an analysis of the Westergard subspace, assuming an elastic material response. This idea is accompanied by the effect of water pressure on the effective (skeleton) stress state. The approach provides any extremely efficient estimate of the depth of the influence zone. The proposed analytical solution is verified against the finite element analysis, assuming that the material response is driven by the modified Cam clay model. The predicted analytical result is found to be in a good agreement with the numerical solution derived with the help of the ADINA 8.1 general-purpose finite element code. The presented results further suggest that this analytical procedure can be applied to solve the interaction problem of the slab and the subsoil and its progress in the structural process, namely by varying the ground water table.
\end{abstract}

Keywords: influence zone, layered subsoil, water pressure, modified Cam clay model, finite element method.

\section{Introduction}

It is an experimentally confirmed fact that a soil changes its material properties substantially when subjected to external loading. When subjected to a certain loading history, the soil alsohas the ability to memorize the highest level of loading mathematically represented by the over-consolidation ratio. In the virgin state, soil deformability is relatively high. By contrast, following the unloading/reloading path there is almost negligible deformation until the highest stress state that the soil has experienced ever before is reached [1], [2]. When using standard recommendations [6] in the design, e.g. in analysing the settlement of foundation subsoil, this soil property is introduced by specifying the depth of the influence zone. It is expected that a reliable estimate of this quantity can follow from a detailed numerical analysis furnished with a suitable constitutive model such as the modified Cam clay model [5]. Such a complex analysis, however, is in direct contradiction with the use of simplified but efficient calculations based on standards. It is therefore imperative to search for an analytical solution that very rapidly provides the required depth of the influence zone. In this paper the general problem is extended to account for the presence of water.

As an example we may consider a foundation slab built inside a deep excavation. If water is present, the construction process usually requires the original water table to be lowered by pumping. After completing the construction stage the pumping is stopped, resulting in a significant change in pore water pressure. Nevertheless, the water table may rise again due to the significant variability of water conditions, which keep changing in the course of the year. This may result in subsequent flooding of the foundation slab. This particular state will be addressed in this paper. To further simplify the complexity of this problem, we limit our attention to a specific case in which the subsoil is first consolidated under dry conditions and subsequently flooded. The effect of this process on the variation of the depth of influence is the main goal of this paper. The numerical analysis is presented to back up the proposed analytical model.

Obviously, for the analytical model to be reliable in describing the soil-structure interaction it is crucial to replace the usual semi-infinite subspace by a layer of finite depth, which is determined by the magnitude of instantaneous loading and the level of previous consolidation. This means that in the case of an analytical analysis of elastic subsoil we are required to replace the known Boussinesq solution by the solution of an elastic layer [3].

To further introduce the subject, consider the distribution of the vertical stresses according to Fig. 1. Due to excavation up to a certain depth $h$ there is a reduction in the original geostatic stress state, which sets the initial "structural strength" of the soil, represented by the pre-consolidation pressure (the highest stress level the soil has experience in the prior loading history [1]). The subsequent surcharge in the footing bottom gives a further redistribution of the vertical stress. In addition, the effective stress state in the soil skeleton is influenced by varying the level of the ground water table. It is assumed that the skeleton deformations are negligible in the region where the vertical stress due to surcharge at the footing bottom combined with the reduced geostatic stress (by excavation and water pressure), exceeds the original geostatic stress. This condition sets the depth of the influence zone $H$.

The paper is organized as follows. The analytical analysis of the influence zone problem is presented in the next section. The case of no water present is considered first. Subsequently, the solution is extended by introducing the effect of water pressure. The geometrical model together with the assumed loading history introduced in the numerical analysis is outlined next. Here, the numerical results are derived with the help of the ADINA 8.1 general-purpose finite element code [7]. The last section compares the numerical and analytical results, and supports the proposed analytical approach. 


\section{Analytical solution}

\section{a) Without the influence of water}

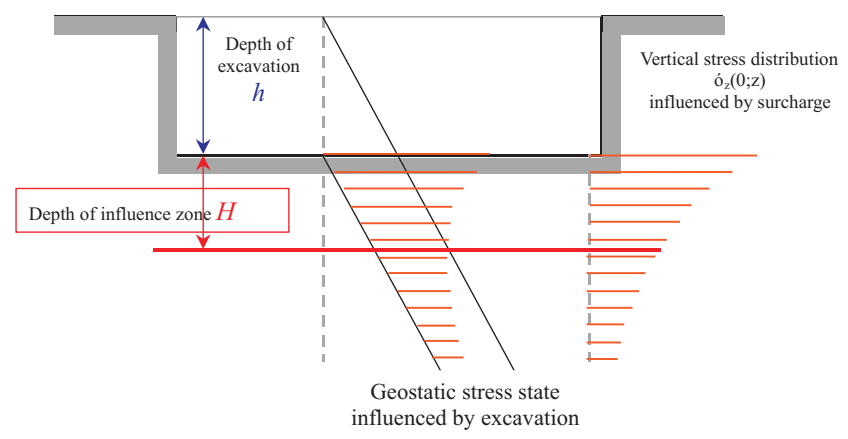

Fig. 1: The basic idea of influence zone analysis

The aim of the analytical solution is to determine the deformation of an elastic layer in the vertical direction. The solution procedure builds on neglecting the horizontal displacements, similar to standard assumptions applied to the analysis of the Westergard subspace. Cleary, such an assumption, resulting in a stiffer soil response, is in a good agreement with reality, owing to the assumption of an "infinite" concrete foundation slab. The problem formulation is evident from Fig. 2. Referring to the Kantorowitch method, the distribution of the displacement field is searched in the form

$$
\begin{aligned}
& w(x, y, z)=\sum_{j=1,3,5}^{\infty} w_{j}(x, y) \psi_{j}(z), \\
& \psi_{j}(z)=\frac{1}{\sqrt{H}} \sin \frac{j \pi}{2 H} z,
\end{aligned}
$$

where $\psi$ is a known function of variable $z$ and represents a complete set of base functions.
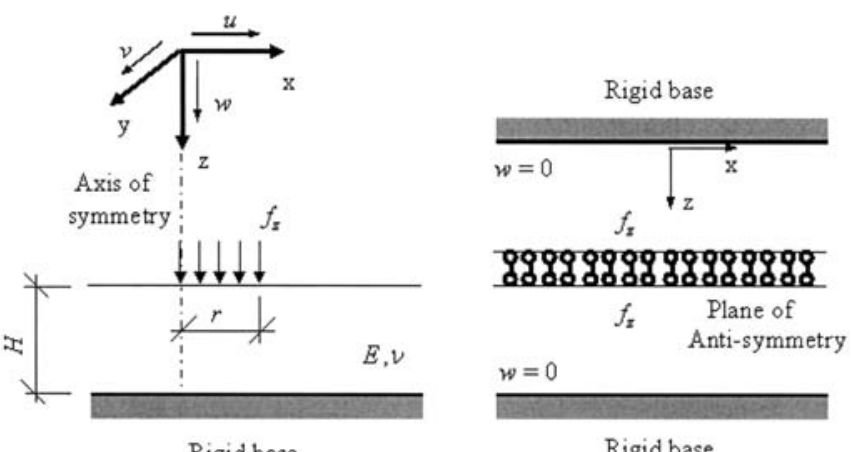

Fig. 2: Coordinate system and plane of anti-symmetry

Invoking the Lagrange principle of virtual work, we have the equilibrium condition in the form of Bessel's differential equation for unknown vertical displacement $w$ as

$$
w_{j, x x}+\frac{1}{x} w_{j, x}-(j \alpha)^{2} w_{j}=(-1)^{\frac{j-1}{2}} \frac{f_{z}}{C_{2}}
$$

accompanied by the following boundary conditions

$$
\begin{aligned}
& w_{j}(\infty)=0, \\
& \left.\frac{\mathrm{d} w_{j}(x)}{\mathrm{d} x}\right|_{x=0}=0 .
\end{aligned}
$$

Assuming a uniform loading $f_{z}$ applied over a circular region of radius $r$, Fig. 2, the known solution receives the form [4]

$x \in\langle 0 ; r\rangle \quad w_{j}(x)={ }^{j} \bar{K}_{1} I_{0}(j \alpha x)+\frac{w_{p 1}}{j^{2}}(-1)^{\frac{j-1}{2}}$,

$x \in\langle r ; \infty\rangle \quad w_{j}(x)={ }^{j} \bar{K}_{2} K_{0}(j \alpha x)$,

where $\alpha=\sqrt{\frac{C_{1}}{C_{2}}}, \quad w_{p 1}=\frac{f_{z}}{C_{1}}, C_{1}=\frac{\sqrt{H} \cdot \pi^{2}}{8 H^{2}} E_{\text {oed }}, C_{2}=\frac{\sqrt{H}}{2} G$; $E_{\text {oed }}, \mathrm{G}$, are the known oedometric and shear modulus.

The two integration constants ${ }^{j} \bar{K}_{1},{ }^{j} \bar{K}_{2}$ are found from the continuity of displacements and their first derivatives at the loading boundary $(x=r)$. After some algebra we arrive at

$$
\begin{aligned}
& j \bar{K}_{1}=-\frac{w_{p 1}}{j}(-1)^{\frac{j-1}{2}} \alpha r K_{1}(j \alpha r), \\
& { }^{j} \bar{K}_{2}=-\frac{w_{p 1}}{j}(-1)^{\frac{j-1}{2}} \alpha r I_{1}(j \alpha r) .
\end{aligned}
$$

Introducing Eq. (4) into Eq. (1) and taking into account

$$
\sum_{n=0}^{\infty}(-1)^{n} \frac{\sin [(2 n+1) z]}{(2 n+1)^{2}}=\frac{\pi \cdot z}{4}
$$

gives the searched vertical displacement as

$x \in\langle 0 ; r\rangle$

$$
w(x, z)=\frac{w_{p 1}}{\sqrt{H}}\left\{\frac{\pi^{2}}{8 H} z-\right.
$$$$
\left.-\sum_{n=0}^{\infty}(-1)^{n} \frac{\alpha r}{2 n+1} K_{1}[(2 n+1) \alpha r] I_{0}[(2 n+1) \alpha x] \sin \left[\frac{(2 n+1) \pi}{2 H} z\right]\right\},
$$

$x \in\langle r ; \infty\rangle$

$w(x, z)=\frac{w_{p 1}}{\sqrt{H}} \times$

$\times\left\{\sum_{n=0}^{\infty}(-1)^{n} \frac{\alpha r}{2 n+1} I_{1}[(2 n+1) \alpha r] K_{0}[(2 n+1) \alpha x] \sin \left[\frac{(2 n+1) \pi}{2 H} z\right]\right\}$.

For the purposes of solution, it is advantageous to increase the domain of interest by introducing a plane of anti-symmetry, as shown in Fig. 2. This step allows the introduction of homogeneous boundary conditions on both bases. Consequently, only the odd terms, $j=2 n+1, n=0,1,2, \ldots$, in series (1) are considered. The vertical stresses $\sigma_{z}$ then follow from the generalized Hooke law and are given by 


$$
\begin{aligned}
& x \in\langle 0 ; r\rangle \\
& \sigma_{z}(x, z)=f_{z}\left\{1-\sqrt{\frac{E_{\text {oed }}}{G}} \frac{2 r}{H} \times\right. \\
& \left.\times \sum_{n=0}^{\infty}(-1)^{n} K_{1}[(2 n+1) \alpha r] I_{0}[(2 n+1) \alpha x] \cos \left[\frac{(2 n+1) \pi}{2 H} z\right]\right\}, \\
& x \in\langle r ; \infty\rangle \\
& \sigma_{z}(x, z)=f_{z} \sqrt{\frac{E_{\text {oed }}}{G}} \frac{2 r}{H} \times \\
& \times\left\{\sum_{n=0}^{\infty}(-1)^{n} I_{1}[(2 n+1) \alpha r] K_{0}[(2 n+1) \alpha x] \cos \left[\frac{(2 n+1) \pi}{2 H} z\right]\right\} . \\
& )
\end{aligned}
$$

For the sake of clarity we further denote

$$
\beta=\sqrt{\frac{E_{\text {oed }}}{G}} \frac{r}{H}=\sqrt{\frac{2-2 v}{1-2 v}} \frac{r}{H} .
$$

Substituting Eq. (8) into Eq. (7) yields $x \in\langle 0 ; r\rangle$

$\sigma_{z}(x, 2 H)=f_{z}\left\{1-2 \beta \sum_{n=0}^{\infty}(-1)^{n} K_{1}[(2 n+1) \alpha r] I_{0}[(2 n+1) \alpha x]\right\}$,

$x \in\langle r ; \infty\rangle$

$\sigma_{z}(x, 2 H)=f_{z} 2 \beta \sum_{n=0}^{\infty}(-1)^{n} I_{1}[(2 n+1) \alpha r] K_{0}[(2 n+1) \alpha x]$.

Focusing on the distribution of vertical stress in the center of loading $x=0$ where the stress reaches its maximum

$$
\sigma_{z}(x, z) \leq \sigma_{z}(0, z),
$$

we get $(x=0)$

$$
\begin{aligned}
& \sigma_{z}(0,2 H)=f_{z}[1-2 F(\beta)] \\
& F(\beta)=\beta \sum_{n=0}^{\infty} K_{1}\left((2 n+1) \beta \frac{\pi}{2}\right) .
\end{aligned}
$$

Referring to Fig. 1, the depth of influence zone $H$ is found at the point where the vertical stress due to surcharge $f_{z}$ becomes equal to the original geostatic stress. Therefore

$$
\sigma_{z}(0,2 H)=\gamma h, \quad F(\beta)=\frac{1}{2}\left(1-\frac{\gamma h}{f_{z}}\right) .
$$

A graphical representation of Eq. (11) is depicted in Fig. 3. As is evident from Fig. 3, the evaluation of $H$ may be carried out in the following way: based on the foundation conditions (known surcharge $f_{z}$, depth of excavation $h$ and specific weight of soil $\gamma$ ), we determine function $F(\beta)$ according to (12). Parameter $\beta$ and consequently depth $H$ follow directly from Fig. 3 . Recall that due to the introduced constraints the resulting value of $H$ will always be larger than the actual value.

\section{b) Extension to account for the influence of water}

This section outlines a straightforward extension of the previous solution for cases when the response of the foundation subsoil is influenced by the presence of water. This step thus requires a superposition of the influence of excavation, surcharge and water pressure, as is evident from Fig. 4 (a).

The decrease in effective stress due to the presence of water can be imagined as a pseudo increase in structural strength (over-consolidation). A graphical interpretation is displayed in Fig. 4(b). To proceed, we first recall identity (11). This equation, when combined with the idea of pseudo-over consolidation, then provides

$$
f_{z}[1-2 F(\beta)]=\gamma h+\gamma_{w}\left(h_{w}+H\right) .
$$

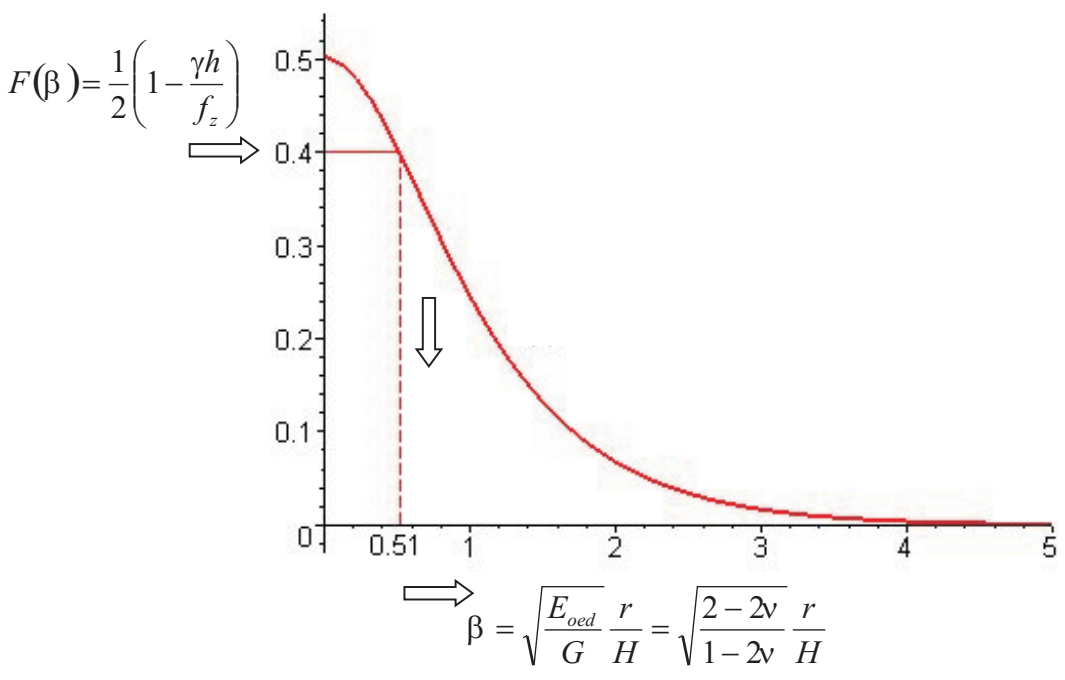

Fig. 3: Determination of $H$ 


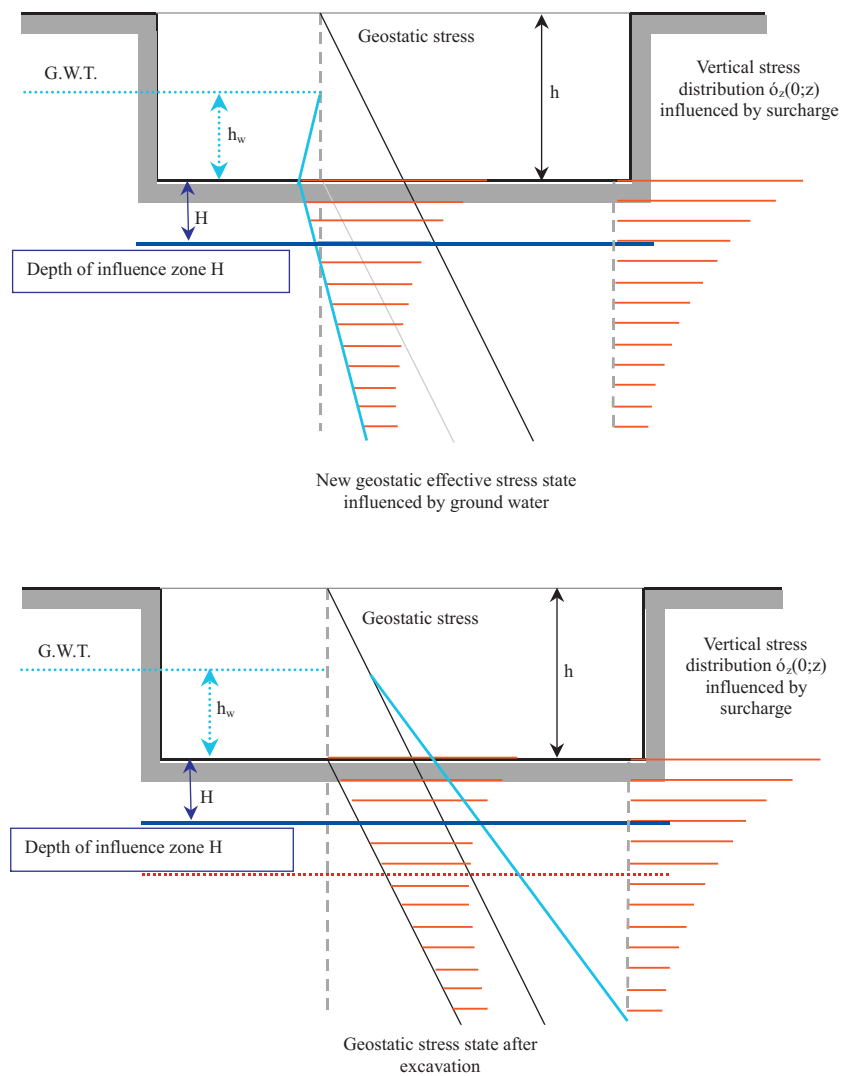

Fig. 4: The basic idea of influence zone analysis: (a) changes in effective stress distribution, (b) pseudo over-consolidation enhancement

From relation (8) we obtain

$$
H=\sqrt{\frac{E_{o e d}}{G}} \frac{r}{\beta}=\sqrt{\frac{2-2 v}{1-2 v}} \frac{r}{\beta} .
$$

Substituting (14) into (13) gives after some manipulation

$\left(1-\frac{\gamma h+\gamma_{w} h_{w}}{f_{z}}\right)=2 F(\beta)+\frac{\gamma_{w}}{f_{z}} \sqrt{\frac{2-2 v}{1-2 v}} \frac{r}{\beta}=G(\beta)$.

Comparing (12) and (15) immediately suggests formal similarity. Thus the calculation of the depth of the influence zone then naturally follows the same footsteps described in the previous section (case with no water present).

First, we introduce the graphical representation of Eq. (15) depicted in Fig. 5 and we proceed as before: based on the foundation conditions (known surcharge $f_{z}$, depth of excavation $h$, specific weight of soil $\gamma$, level of ground water table $h_{w}$ and the specific weight of water) we determine the value of function $G(\beta)$ according to (15). In contrast with the dry case, the formula for function $G(\beta)$ further depends on the Poisson ratio $v$ of the soil. Parameter $\beta$ and consequently depth $H$ follow directly from Fig. 5. Once again, due to the introduced constraints, the resulting value of $H$ will always be larger than the actual value.

\section{Numerical solution}

As mentioned in the introductory part, the depth of the influence zone can be estimated by combining finite element analysis with a suitable constitutive model. It appears that a suitable candidate can be found from a group of models capable of representing the soil memory, such as critical state models. A critical state model is a form of an isotropic strain-hardening (softening) law. It introduces a distinction between yielding and ultimate collapse by using the concept of a critical state line in conjunction with a strain-dependent yield surface. The yield surface therefore expands or contracts as the soil hardens or softens. Such a soil behavior is well described by the modified Cam clay model [5]. Here, the initial size of the yield surface is governed by the maximum

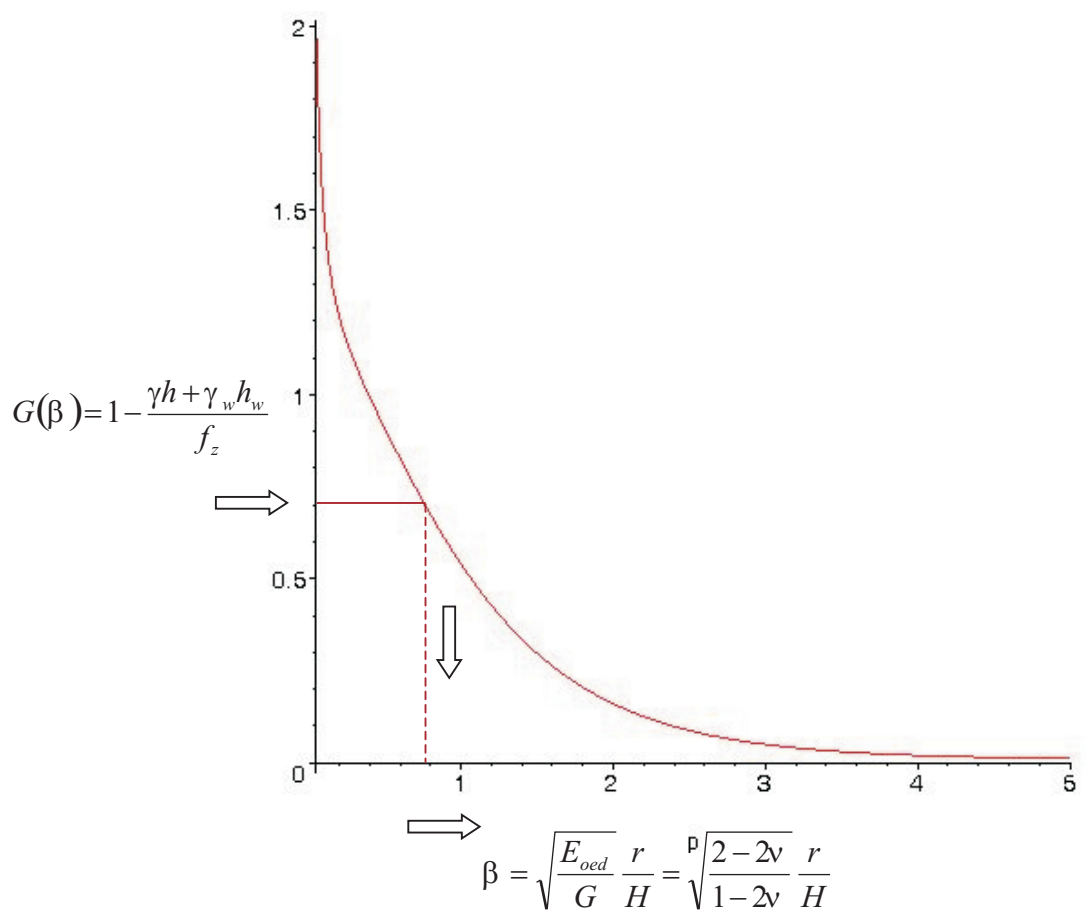

Fig. 5: Determination of $H$ 
SOIL CONSOLIDATION LOAD

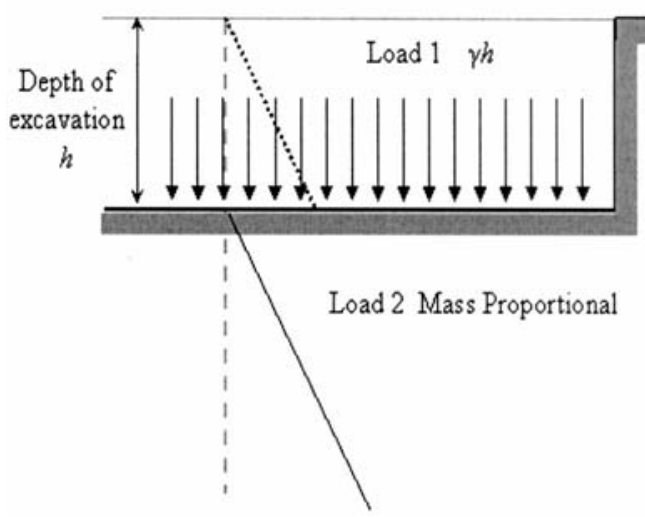

Fig. 6 (a) Modeling the initial state, (b) reloading stage

pre-consolidation pressure to which the soil has previously been subjected during its past history, thus representing the memory effect.

A geometrical model for the finite element analysis, together with the assumed loading sequence, appears in Figs. 6a, b. Note that the bottom boundary of the model was placed at a depth of $5 \mathrm{~m}$. The original geostatic stress state was developed in several steps assuming that the material point moves down the normal consolidation line. This was found from a superposition of the stress after excavation and the surcharge applied at the footing bottom over the total span of the geometrical mode, and with the magnitude corresponding to the amount of excavated soil. In the second stage this amount of loading was removed in order to simulate the unloading due to excavation, thus building the new reduced stress state Fig. 6a, recall also Fig. 1. Finally a new surcharge at the footing bottom was supplied, Fig. 6b, to represent the effect of the upper structure. Such a loading / unloading sequence corresponds exactly to the loading conditions ap-

\section{FINAL LOADING}

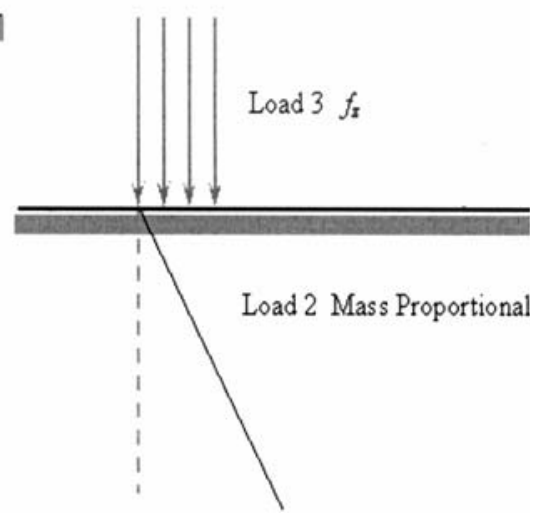

plied in the analytical analysis. The present axisymmetric problem was solved with the help of the finite element code ADINA 8.1.

The material parameters of the modified Cam clay model and the value of the secant oedometric modulus $E_{\text {oed }}$ used in the analytical analysis appear in Table 1 . The slope of the NCL line $\lambda$ was chosen such as to approximately correspond to $E_{\text {oed }}$. The slope of the swelling line was taken as $1 / 10 \lambda$.

The set of results corresponds to the depth of excavation $h=5 \mathrm{~m}$, to various magnitudes of the applied surcharge $f_{z}$ and to several levels of ground water tables. The plots showing the results appear in Figs. 7-11. Note that the dashed

Table 1: Cam clay parameters

\begin{tabular}{|c|c|c|c|c|c|c|c|c|}
\hline $\begin{array}{c}E_{\text {oed }} \\
{[\mathrm{kPa}]}\end{array}$ & $v$ & $\begin{array}{c}\gamma \\
{\left[\mathrm{kN} / \mathrm{m}^{3}\right]}\end{array}$ & $\lambda$ & $\kappa$ & $K_{0}$ & $M$ & OCR & $\Gamma$ \\
\hline 42860 & 0.4 & 20 & 0.005 & 0.0005 & 1 & 1.2 & 1 & 1.2 \\
\hline
\end{tabular}

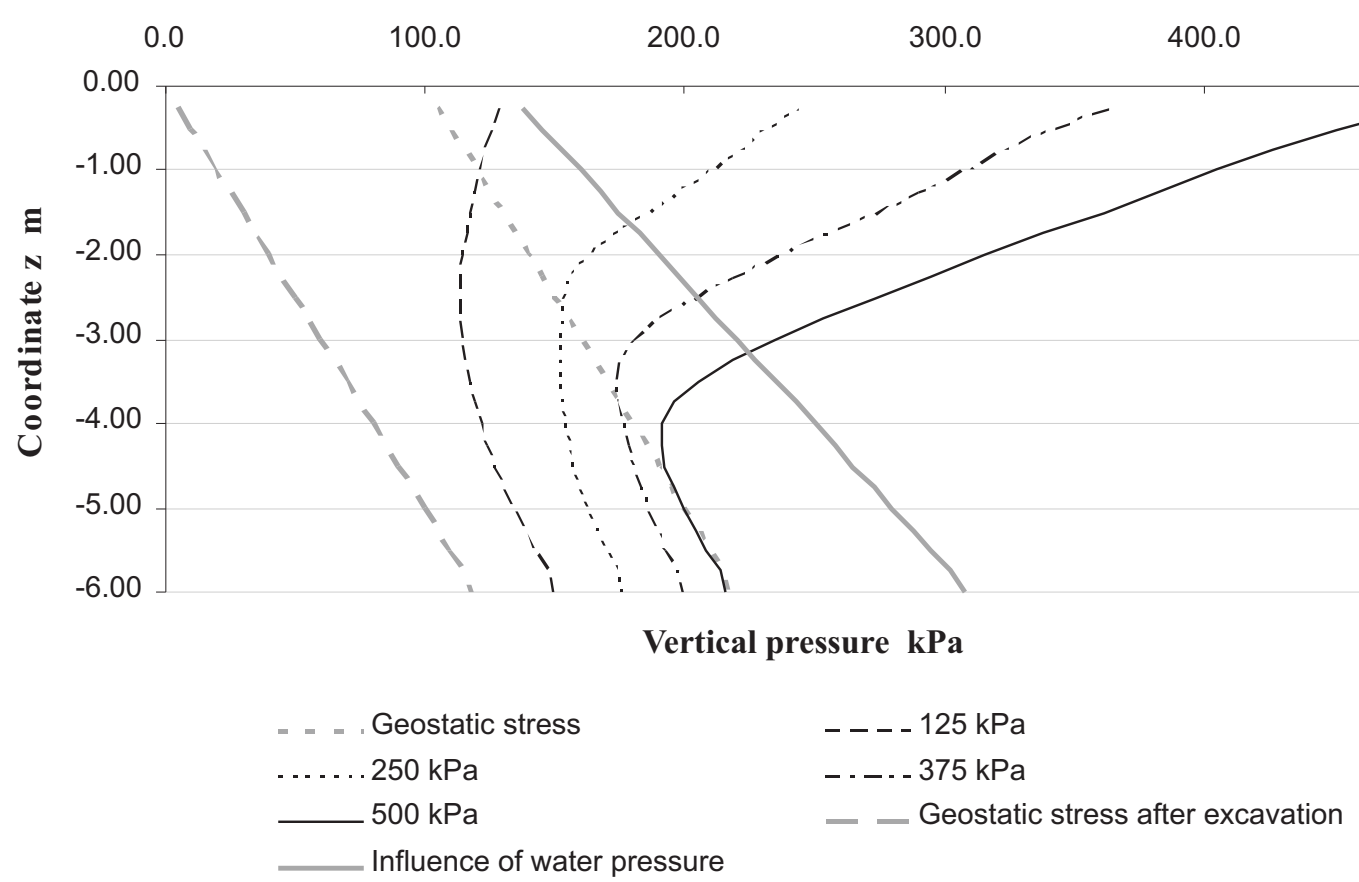

Fig. 7: Change in the depth of the influence zone due to the level of surcharge ( $5 \mathrm{~m}$ excavation), $1 \mathrm{D}$ analysis - horizontal displacements are fixed (analysis compatible with analytical solution) 
straight line represents the distribution of the geostatic stress after excavation, while the solid straight line corresponds to the initial geostatic stress enhanced by G.W.T. (see Fig. 4b).
Clearly, the depth of the influence zone is found as a point of intersection of the final stress distribution with the solid line.

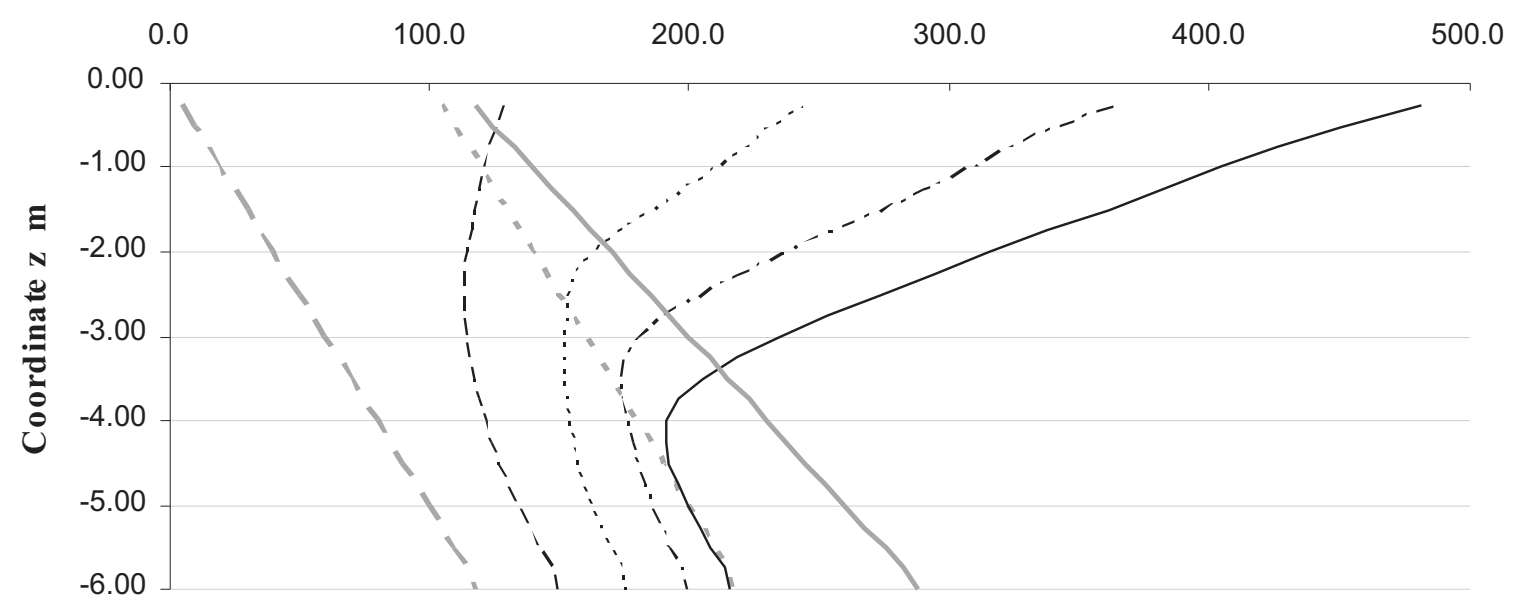

Vertical pressure $\mathrm{kPa}$
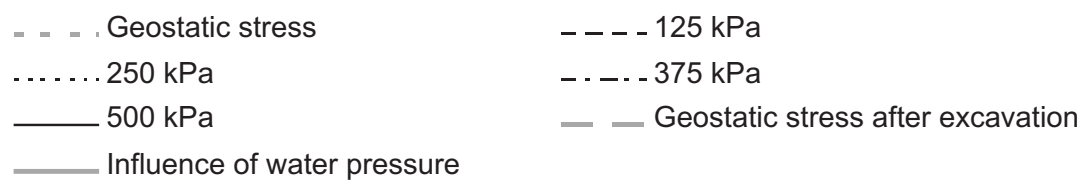

Fig. 8: Change in the depth of the influence zone due to the change in Poisson's ratio (5 m excavation), horizontal displacements are fixed

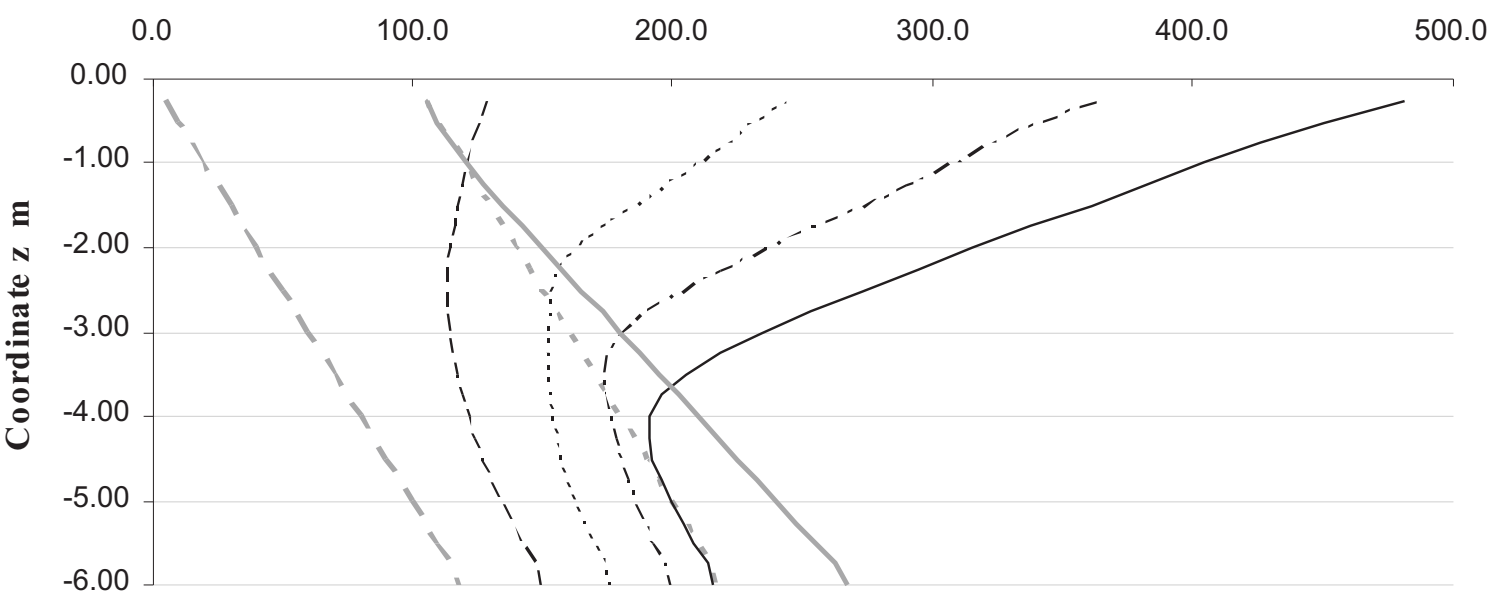

Vertical pressure $\mathrm{kPa}$

\begin{tabular}{|c|c|}
\hline$=-$ - Geostatic stress & $---125 \mathrm{kPa}$ \\
\hline$\ldots \ldots 250 \mathrm{kPa}$ & -... $375 \mathrm{kPa}$ \\
\hline$-500 \mathrm{kPa}$ & - - Geostatic stress after excavation \\
\hline
\end{tabular}

Fig. 9: Comparison between the simplified 1D analysis (horizontal displacements are fixed) and full 2D axisymmetric analysis (5 m excavation) 


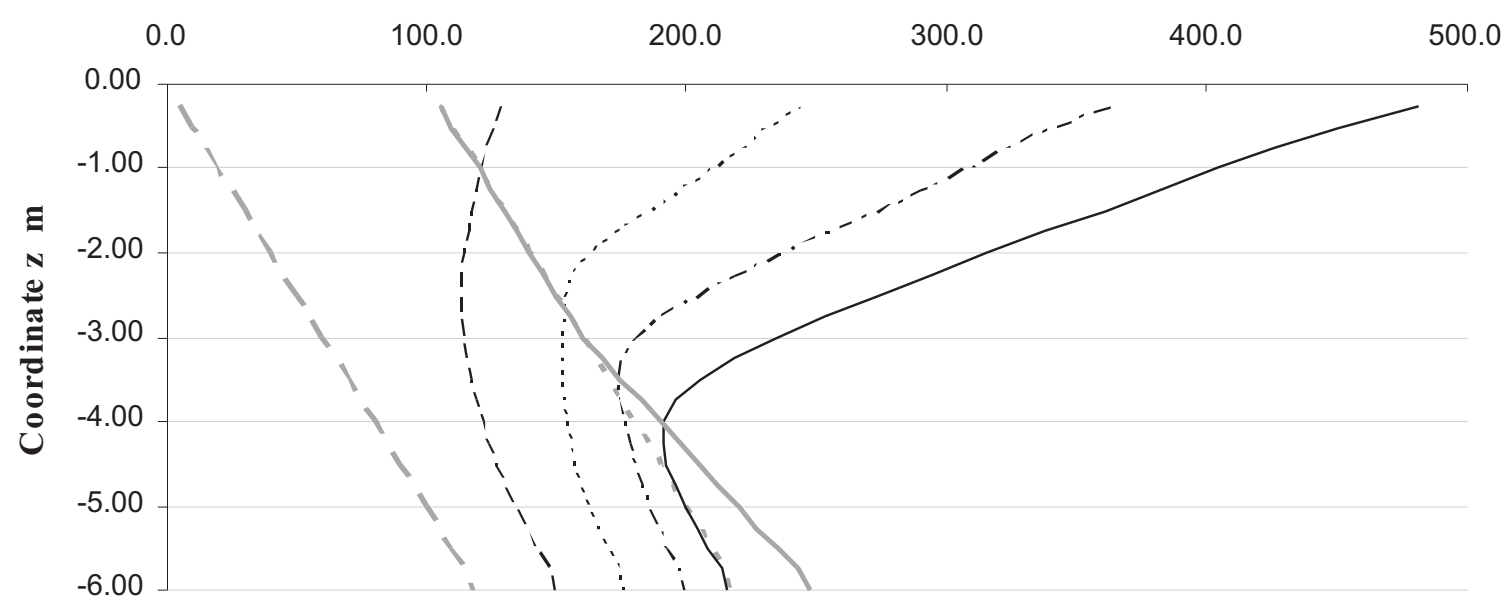

Vertical pressure $\mathrm{kPa}$

\begin{tabular}{l}
$=-$ Geostatic stress \\
$\ldots . . .250 \mathrm{kPa}$ \\
\hline Influence of water pressure
\end{tabular}

$$
\begin{aligned}
& ---125 \mathrm{kPa} \\
& -\cdot-\cdot 375 \mathrm{kPa} \\
& -\quad-\text { Geostatic stress after excavation }
\end{aligned}
$$

Fig. 10: Change in the depth of the influence zone due to the level of surcharge ( $4 \mathrm{~m}$ excavation), $1 \mathrm{D}$ analysis - horizontal displacements are fixed

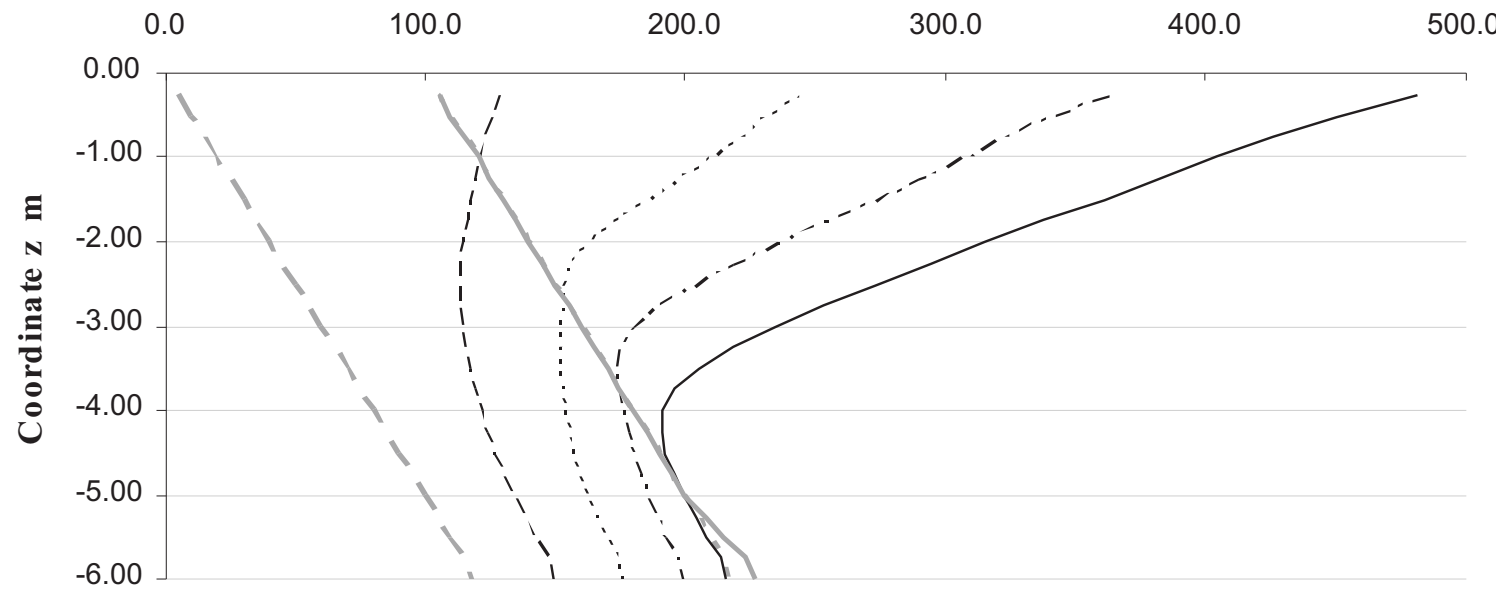

Vertical pressure $\mathrm{kPa}$

\begin{tabular}{l}
$=\ldots$ Geostatic stress \\
$\ldots \ldots 250 \mathrm{kPa}$ \\
\hline Influence of water pressure
\end{tabular}

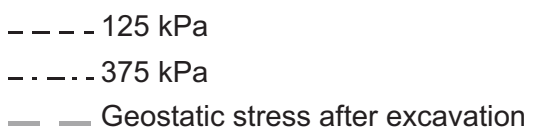

Fig. 11: Change in the depth of the influence zone due to the level of surcharge (3 m excavation), 1D analysis - horizontal displacements are fixed

\section{Comparison of analytical and numerical results}

Comparing the values of $H$ derived from both analytical and numerical analysis, Table 2-3 shows good agreement, thus supporting the applicability of the proposed analytical solution. However, care must be taken as the solution somewhat overestimates the depth of $H$, fortunately on the safe side, due to the constraints that were introduced.

Table 2: Analytical solution of the influence zone depth for various levels of G.W.T.

\begin{tabular}{|c|c|c|c|c|c|}
\hline $\begin{array}{c}f_{\mathrm{Z}} \\
{[\mathrm{kPa}]}\end{array}$ & $\begin{array}{c}\text { G.W.T. }= \\
=3 \mathrm{~m}\end{array}$ & $\begin{array}{c}\text { G.W.T. }= \\
=1 \mathrm{~m}\end{array}$ & $\begin{array}{c}\text { G.W.T. }= \\
=-1 \mathrm{~m}\end{array}$ & $\begin{array}{c}\text { G.W.T. }= \\
=-3 \mathrm{~m}\end{array}$ & $\begin{array}{c}\text { G.W.T. }= \\
=-5 \mathrm{~m}\end{array}$ \\
\hline 125 & & 0.875 & 1.35 & 1.35 & 1.35 \\
\hline 250 & 2.05 & 2.35 & 2.65 & 2.99 & 2.99 \\
\hline 375 & 2.93 & 3.13 & 3.43 & 3.78 & 4.19 \\
\hline 500 & 3.41 & 3.72 & 4.03 & 4.39 & 4.76 \\
\hline
\end{tabular}


Table 3: Numerical solution of the influence zone depth for various levels of G.W.T.

\begin{tabular}{|c|c|c|c|c|c|}
\hline $\begin{array}{c}f_{z} \\
{[\mathrm{kPa}]}\end{array}$ & $\begin{array}{c}\text { G.W.T. } \\
=3 \mathrm{~m}\end{array}$ & $\begin{array}{c}\text { G.W.T. }= \\
=1 \mathrm{~m}\end{array}$ & $\begin{array}{c}\text { G.W.T. }= \\
=-1 \mathrm{~m}\end{array}$ & $\begin{array}{c}\text { G.W.T. }= \\
=-3 \mathrm{~m}\end{array}$ & $\begin{array}{c}\text { G.W.T. }= \\
=-5 \mathrm{~m}\end{array}$ \\
\hline 125 & & 0.5 & 1.0 & 1.0 & 1.0 \\
\hline 250 & 1.75 & 2.0 & 2.25 & 2.6 & 2.6 \\
\hline 375 & 2.5 & 2.75 & 3.0 & 3.5 & 3.75 \\
\hline 500 & 3.25 & 3.4 & 3.7 & 4.0 & 4.75 \\
\hline
\end{tabular}

\section{Conclusions}

It was shown that an extremely rapid estimate of the depth of influence zone can be provided by an analytical solution of the problem of deformation of an elastic layer. The applicability of the proposed approach was further confirmed by the numerical analysis using the FE method combined with the modified Cam clay model. The assumption that vertical displacement can be neglected is in good agreement with the conditions inside the subsoil below the foundations slabs. It was shown that flooding of the foundation base results in a decrease in the depth of the influence zone. It is expected, however, that the initial step associated with lowering the ground water table by pumping, not considered here, may lead to a larger extent of the influenced zone. This point is under current investigation. In addition, several experiments are new being carried out to verify the proposed theory.

Finally, it is worth to mentioned that lowering the depth of the influence zone (flooding) reduces the effect of shear in the subsoil and leads to a more or less uniform distribution of the stress along the foundation base. As a direct consequence, there is an increase in field moments. The increase in the moments is further magnified by uplift of water. If this water effect is not taken into account in the construction design, we may anticipate an increase in the current state of damage and consequently seepage of water through the foundation slab.

\section{Acknowledgments}

Support from GAČR 103/04/1134 is gratefully acknowledged.

\section{References}

[1] Bowles J. E.: "Foundation analysis and design." New York, McGraw-Hill, 1966.

[2] Kuklík P., Šejnoha M., Mareš J.: "The structural strength of soil from the isotropic consolidation point of view." In: Computational Mechanics for the Next Millenium, Volume 2, Proceedings of APCOM 99 (Editors: Wang C. M., Lee, K. H., Ang, K.K.), Singapore, Elsevier 1999, p. 797-802.

[3] Kuklík P., Kopáčková M.: "Comparison of elastic layer solution with Boussinesq half space solution. (in Czech)." In: Stavebni Obzor, Prague, CTU in Prague, in print.

[4] Rektorys K.: „Přehled užité matematiky I.“ Sixth edition, Praha, ČMT Prometheus 1995.

[5] Lewis R. W., Schrefler B. A.: "The finite element method in the static and dynamic deformation and consolidation of porous media." Chichester, John Wiley \& Sons Ltd, England 1998, p. 75-143.

[6] ČSN P ENV 1997-1 (EUROCOD 7) „Navrhování geotechnických konstrukcí.“ Praha 1996.

[7] ADINA 8.1, ADINA R \& D, Inc., Watertown, USA, 2004.

Doc. Ing. Pavel Kuklík, CSc.

phone: +420224354486

e-mail: kuklikpa@fsv.cvut.cz

Department of Structural Mechanics

RNDr. Marie Kopáčková, CSc. phone: +420224354385

e-mail: marie.kopackova@fsv.cvut.cz

Department of Mathematics

Czech Technical University in Prague

Faculty of Civil Engineering

Thákurova 7

166 29, Praha 6, Czech Republic 